Review

\title{
Pressure Sensor Based on the Fiber-Optic Extrinsic Fabry-Perot Interferometer
}

\author{
Qingxu YU and Xinlei ZHOU \\ School of Physics and Optoelectronic Technology, Dalian University of Technology, Dalian, 116023, P.R. China \\ *Corresponding author: Qingxu YUＥ-mail: yuqx@dlut.edu.cn
}

\begin{abstract}
Pressure sensors based on fiber-optic extrinsic Fabry-Perot interferometer (EFPI) have been extensively applied in various industrial and biomedical fields. In this paper, some key improvements of EFPI-based pressure sensors such as the controlled thermal bonding technique, diaphragm-based EFPI sensors, and white light interference technology have been reviewed. Recent progress on signal demodulation method and applications of EFPI-based pressure sensors has been introduced. Signal demodulation algorithms based on the cross correlation and mean square error (MSE) estimation have been proposed for retrieving the cavity length of EFPI. Absolute measurement with a resolution of $0.08 \mathrm{~nm}$ over large dynamic range has been carried out. For downhole monitoring, an EFPI and a fiber Bragg grating (FBG) cascade multiplexing fiber-optic sensor system has been developed, which can operate in temperature $300{ }^{\circ} \mathrm{C}$ with a good long-term stability and extremely low temperature cross-sensitivity. Diaphragm-based EFPI pressure sensors have been successfully used for low pressure and acoustic wave detection. Experimental results show that a sensitivity of $31 \mathrm{mV} / \mathrm{Pa}$ in the frequency range of $100 \mathrm{~Hz}$ to $12.7 \mathrm{kHz}$ for aeroacoustic wave detection has been obtained.
\end{abstract}

Keywords: Pressure measurement, Fabry-Perot interferometer, signal demodulation, downhole monitoring, fiber acoustic sensor

\section{Introduction}

High-performance measurement on static and dynamic pressure is extremely important in many industrial areas, such as petrochemical industry, fluid engineering, wind tunnel test, biomedicine, and industrial safety. For example, reliable pressure measurement of the underground oil reservoir can provide key data which can be used to determine the quantity of oil reserve and to optimize the production rates of reservoir recovery $[1,2]$. However, many of these industrial processes involve harsh environment, such as high temperature, chemical corrosion, strong electromagnetic interference (EMI), and high-energy radiation exposure. These extreme physical conditions often lead to conventional pressure sensors very difficult to apply and incapable of meeting current and future measurement demands. This situation suggests that innovative sensors capable of operating in various harsh environments should be developed to support industrial efforts.

Fiber-optic sensors have been demonstrated to be especially attractive for the measurement of a wide variety of physical and chemical parameters in harsh environment in past years [3-6]. In comparison with conventional sensors, fiber-optic sensors own many advantages, such as immunity to

Received: 30 Septemper 2010 / Revised version: 14 October 2010

CThe Authors(s) 2010.This article is published with open access at Springerlink.com 
EMI, small size, light weight, resistance to chemical corrosion, high accuracy, resolution, and capability of remote operation. These advantages have promoted worldwide research activities in the field of optical fiber sensor technologies for harsh environment. In recent years, fiber-optic based pressure sensors employing various mechanisms have been reported, including microbending [7], photoelastics [8-10], and fiber gratings sensor [11-13].

Since the extrinsic Fabry-Perot interferometer (EFPI) based fiber-optic sensor was presented by Kent A. Murphy in 1991 [14], EFPI has been developed into pressure sensor and drawn a great deal of research interests due to the advantages of smaller size, immunity to the polarization-induced fading, and extremely high sensitivity. In this paper, a review on EFPI-based pressure sensors has been given. Firstly, major progresses of EFPI-based sensors in past years are reviewed. Then, the basic sensing mechanism of EFPI-based pressure sensor is described and various signal demodulation methods are summarized. Finally, application progress of EFPI-based pressure sensor, including downhole monitoring, liquid-level monitoring, acoustic wave detection, and trace gas detection, are introduced.

\section{Review of major improvements of EFPI-based pressure sensors in past years}

\subsection{Controllable thermal bonding technique}

EFPI-based pressure sensors have been usually fabricated by inserting two endface-cleaved fibers into an alignment capillary tube with a proper inner diameter [15], as shown in Fig. 1. In the early work on EFPI-based pressure sensors, epoxies were applied to bond the fiber with the alignment capillary tube. However, sensors fabricated with this method have some disadvantages which limit their use in harsh environment. For example, epoxy-based sensors have a bad performance in terms of thermal stability and mechanical strength. The working temperature of epoxy-based sensors is also limited by that of the epoxy. In addition, because of inherent thermal expansion coefficient difference between epoxy and fiber, epoxy-based sensors are usually sensitive to temperature change, which would cause a severe temperature-pressure cross-sensitivity problem. To overcome these drawbacks, Anbo Wang et al. presented a novel sensor fabrication system based on the controlled thermal bonding method [4, 16]. They successfully used a $\mathrm{CO}_{2}$ laser to thermally fuse the fiber and the capillary tube together permanently. EFPI-based sensors fabricated by this method have the advantages of good long-term stability and low temperature-pressure cross-sensitivity. This epoxy-free EFPI-based pressure sensors are competent for the industrial application with high temperature and high pressure environment [4-6].

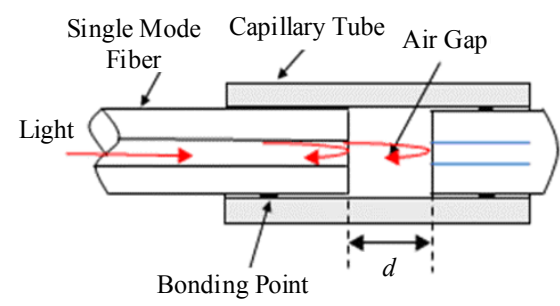

Fig. 1 Typical structure of EFPI-based pressure sensor.

\subsection{Diaphragm-based EFPI pressure sensors}

EFPI-based pressure sensors with the structure shown in Fig. 1 are suitable for static and high pressure measurement, but insensitive to low pressure variation. In order to detect dynamic and low pressure variations, diaphragm-based EFPI (DEFPI) fiber-optic sensor has been proposed [17, 18]. As shown in Fig. 2, DEFPI-based pressure sensors are usually fabricated by manufacturing a microstructure at the fiber end. The manufacturing processes mostly base on micro-electro-mechanical system (MEMS) technologies, such as lithography, chemical wet etching [19], anodic bonding [20], and the organic membrane technology $[21,22]$. 
Fabry-Perot cavity is usually formed between the fiber endface and the inner surface of the diaphragm. The sensitivity and dynamic range of DEFPI-based sensor can be adjusted by changing the diameter, thickness, and/or material of the diaphragm to meet micro and dynamic pressure measurement requirements in industrial and biomedical applications.

Due to possessing advantages of high sensitivity to micro-pressure, fast response, wide bandwidth, lightweight, long life time, and immunity to EMI, DEFPI-based pressure sensors have drawn great research interests and been widely applied in industrial and biomedical fields [23-25].

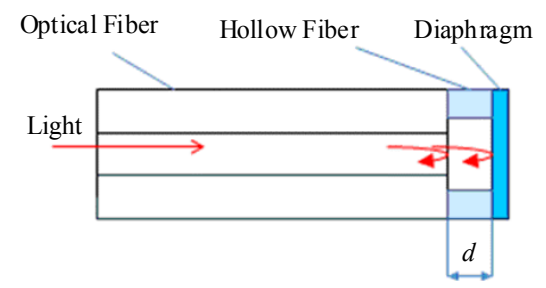

Fig. 2 Typical structure of DEFPI-based pressure sensor.

\subsection{White light based EFPI-sensor}

As for EFPI-based sensors, the measurands are usually retrieved using a specific demodulation scheme through the detection of the reflection spectrum change of Fabry-Perot cavity caused by variations of the measurands. For early EFPI-based sensor systems, a coherent light source was used to achieve a large coherent length, which in turn produced a large dynamic range. For this type of system, the most commonly used signal demodulation method is fringe counting [26]. However, the fringe counting method suffers from problems such as fringe direction ambiguity and low resolution which is of the same order of magnitude as the source wavelength. To solve the ambiguity problem, the low-coherence light (or white light) is employed to retrieve the cavity length of EFPI-based sensor [27-29]. White-light EFPI sensors have many advantages such as immunity to light source power and central wavelength drift or attenuation changes of fiber, high resolution, and absolute measurements. Numerous applications of white-light EFPI sensor systems have been reported, covering a wide range from single point measurement to distributed sensor systems [30-32].

\section{Theory}

\subsection{Mechanism of EFPI-based pressure sensor}

The basic configuration of EFPI-based pressure sensor system is illustrated in Fig. 3. The system includes a light source (usually a white-light source), a spectrometer, an optical coupler, and a sensor head. The sensor head can be a capillary-based EFPI structure (Fig. 1) for static and high pressure measurement or a diaphragm-based EFPI structure (Fig. 2) for micro and dynamic pressure measurement.

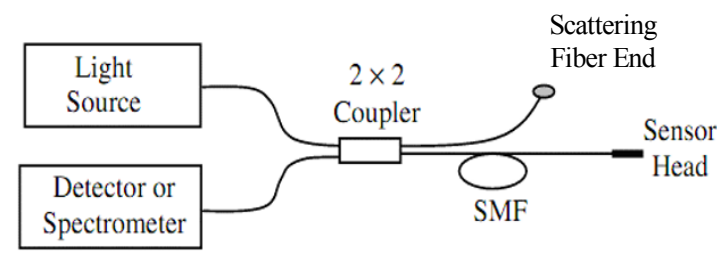

Fig. 3 Basic configuration of EFPI-based pressure sensor system.

The light from light source is launched into a $50 \%$ fiber coupler and propagates along the lead-in/out fiber to the sensor head which is a low-finesse Fabry-Perot interferometer formed by the end-faces of lead-in/out fiber and a reflecting fiber (or a diaphragm). A fraction of the incident light is firstly reflected (about $4 \%$ ) at the endface of lead-in/out fiber and returns directly back to the fiber. The remainder of the light propagates across the air gap to the endface of the reflecting fiber (or the inner surface of the diaphragm), and then the reflected light is recoupled into lead-in/out fiber and interferes with the first reflected light. The output interference signal can be described by [33]

$$
I=2 I_{s}(\lambda)[1+\gamma \cos (\varphi)]
$$

where $I_{s}(\lambda)$ is the intensity distribution of the 
light source, $\gamma$ is the fringe visibility of EFPI, $\varphi$ is the round-trip phase shift, given by

$$
\varphi=\frac{4 \pi d}{\lambda}
$$

where $d$ is the cavity length.

For the sensor geometry shown in Fig. 1, when a pressure $p$ is applied to the sensor head, due to the longitudinal compression of the alignment tube, the cavity length of EFPI will be changed, which can be expressed as [34]

$$
\Delta d=\frac{L r_{o}^{2}}{E r_{o}^{2}-r_{i}^{2}}(1-2 \mu) p
$$

where $E$ is Young's modulus of the tube material, $\mu$ is poisson ratio, $L$ is the distance between two thermal fusion points, $r_{o}$ and $r_{i}$ are outer and inner radii of the capillary tube, respectively. Equation (3) indicates that the cavity length change is linear with respect to the applied pressure.

Equations (1) - (3) reveal that the pressure acting on EFPI sensor can be retrieved by analyzing variations of the interference spectrum through some specific signal processing algorithms, which will be described in the following section.

\subsection{Signal demodulation method}

The signal demodulation algorithms of EFPI-based sensors can be classified into two major categories as linear demodulation [35] (or quadrature demodulation) and spectral detection based demodulation.

\subsubsection{Linear demodulation}

In the linear demodulation, the operation of EFPI-based sensor is limited to a small linear region of the interference fringes near a quadrature point ( $Q$-point) as shown in Fig. 4. In this linear region, the sensor's sensitivity is maximized and can be treated as a constant. However, for the linear demodulation based on the intensity modulation, the measured results are easily influenced by the fluctuations of optical source power and the transmission loss of fiber. To overcome this shortcoming, Anbo Wang et al. developed a self-calibrated interferometric/intensity-based (SCIIB) fiber optic sensor system [36]. SCIIB system uses optical filtering to process optical signal output from Fabry-Perot cavity, so that two signals are produced: one contains information about the cavity displacement and the other contains information regarding undesired effects of the first signal, such as fluctuations of the optical source power, or changes of optical fiber loss. The second signal is used as a reference to eliminate these undesired effects and provides self-calibration functions in the sensor. Based on SCIIB system, Guiju Zhang et al. proposed an improved self-compensated demodulation algorithm [37]. By setting the initial cavity length to a special point at which the interferometric signals from broadband and narrowband channels show reversed phase, more effective compensation and higher resolution of the sensing system are achieved.

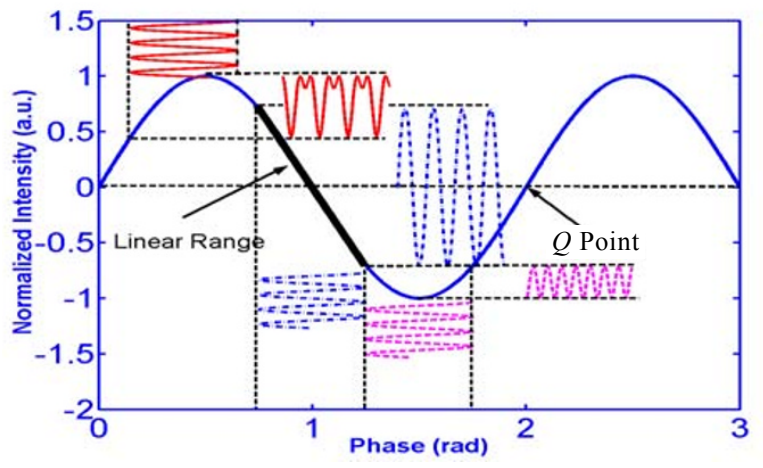

Fig. 4 Schematic diagram of the linear demodulation.

The linear demodulation method has a number of advantages such as high sensitivity, fast signal response, no sensitivity reduction, simple signal processing, and inherently low cost. These features make the linear demodulation particularly be used in dynamic measurement of small perturbations, such as the detection of ultrasonic [38-40], vibration [41], partial discharge [42], and dynamic strain [34]. The drawbacks of operating with the linear demodulation method are limited dynamic range and the challenge for the static operating point control or 
stabilization [39, 43].

\subsubsection{Spectral detection based demodulation method}

As to spectral detection demodulation approach, a spectrometer with a charge-coupled device (CCD) array is usually used to record the reflecting spectrum of EFPI-based sensor and a personal computer is employed for signal processing. A reflecting spectrum of EFPI-based sensor is shown in Fig. 5. According to (1) and (2), when the cavity length $d$ of EFPI changes, the spectrum will have a shift and the frequency of interference fringe will change in the wavelength domain, correspondingly. Therefore, the cavity length can be demodulated by tracking the shift of the spectrum or measuring the frequency variation of interference fringe. Accordingly, signal processing methods such as wavelength-tracking [44], two-peak wavelength interrogation [33], and Fourier transform [45] have been widely used to calculate the cavity length of EFPI-based sensor. Nevertheless, all these methods have some shortcomings. The wavelength-tracking method can not carry out absolute measurement. Two-peak wavelength interrogation method and Fourier transform method usually have a resolution no better than tens of nanometers.

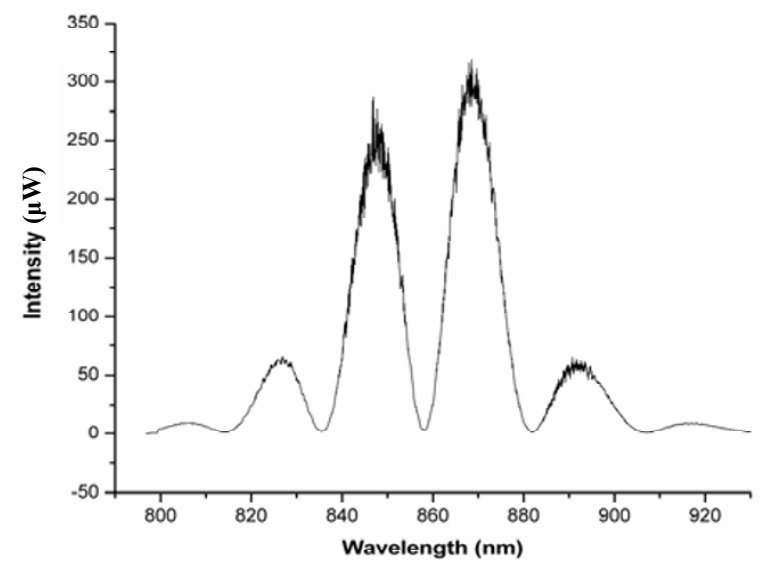

Fig. 5 Interference spectrum from an EFPI sensor.

In order to improve measurement resolution and carry out absolute measurement over a large dynamic range, Zhenguo Jing et al. [46] presented a demodulating method for EFPI sensor based on the cross correlation algorithm, which is capable of providing high resolution up to $0.25 \mathrm{~nm}$ and implementing absolute measurement. Side Song [47] proposed a demodulation algorithm based on mean square error (MSE) estimation for EFPI sensor. MES-based signal processing method introduces MES to estimate the parameter to be measured, which can be expressed as

$$
m s e=\operatorname{var}(\hat{\theta})+\operatorname{bias}^{2}(\hat{\theta})
$$

where $\hat{\theta}$ is an estimated value of the true value $\theta$, $\operatorname{var}(\hat{\theta})$ and $\operatorname{bias}(\hat{\theta})$ are the variance and the deviation of $\hat{\theta}$, respectively.

Equation (4) shows that MSE includes errors caused by the variance and the deviation of $\hat{\theta}$. Only when both $\operatorname{var}(\hat{\theta})$ and $\operatorname{bias}(\hat{\theta})$ are minimum, MSE is minimum. So MSE is an effective parameter to estimate $\hat{\theta}$. Assuming that there is a series of estimated value $\{\hat{\theta}\}$, the $\hat{\theta}$ with the minimum MSE is the best one to represent the true value. MSE-based signal processing method has also the advantages of high resolution up to $0.18 \mathrm{~nm}$ and absolute measurement of the cavity length over a large dynamic range.

\section{Applications of EFPI-based pressure sensor}

\subsection{Downhole monitoring}

Data published by the U. S. Department of Energy indicate that approximately two-thirds of the oil discovered in the U. S. remains in the ground after primary, secondary, and tertiary recovery operations have been completed. This is largely due to the limited availability of information concerning reservoirs, well operation, and well-to-well interrelations. Continuous reliable downhole pressure measurements in wells will provide key data that can permit better, faster reservoir characterization and improving forecasting of reservoir capability, thereby permit operators to optimize the recovery of reserves. Presently, many commercial products based on the fiber-optic sensors for single point pressure measurement in 
wells have been developed. For example, fiber-optic pressure sensors developed by CiDRA corporation can achieve a resolution of $2.06 \mathrm{kPa}$ over the measurement range of $0-103 \mathrm{MPa}$. It can operate in temperature range of $25{ }^{\circ} \mathrm{C}-175{ }^{\circ} \mathrm{C}$. The borehole pressure/temperature (BHPt) series pressure sensor developed by Sabeus corporation can operate in $200{ }^{\circ} \mathrm{C}$ continuously with a resolution of $0.69 \mathrm{kPa}$. In additional, oilfield service companies such as Weatherford, Sabeus, and SENSORNET also have their products for pressure measurement in oil well. And these products have been successfully applied to downhole monitoring. However, as the increasing application of offshore drilling and horizontal wells, pressure sensors that capable of working in higher temperature with good performance such as high resolution, high repeatability, high stability, and low temperature-pressure cross-sensitivity are urgently desired.

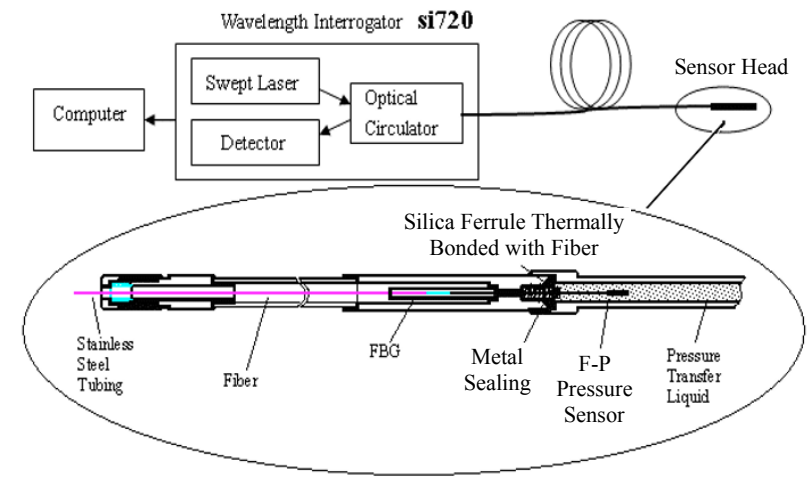

Fig. 6 Configuration of EFPI-FBG multiplexing sensor system.

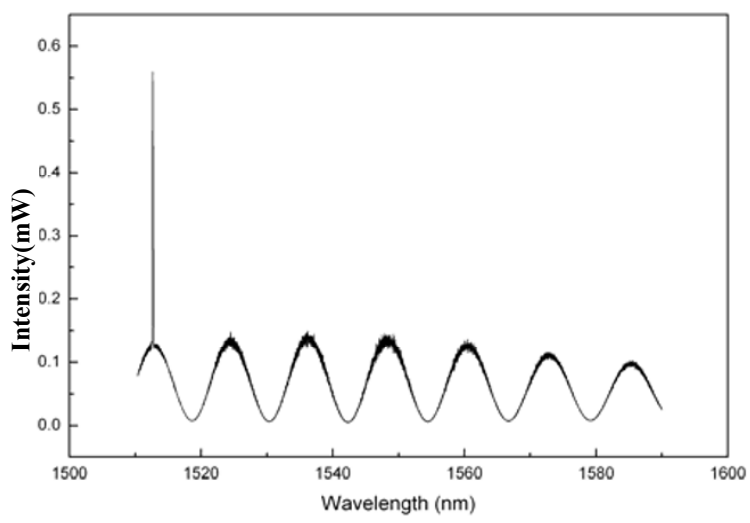

Fig. 7 Reflection spectrum of EFPI-FBG multiplexed sensor.

In recent years, our research group has done a lot of original work on EFPI-based pressure sensor for downhole monitoring. To realize multi-parameters measurement, an EFPI and a fiber Bragg grating (FBG) cascade multiplexing fiber-optic sensor system have been developed [48]. The configuration of EFPI-FBG sensor system and the reflection spectrum of EFPI-FBG sensor head are shown in Figs. 6 and 7, respectively.

In addition to capable of measuring temperature and pressure simultaneously, the temperature-pressure cross-sensitivity problem can be effectively solved by using a temperature compensation calculation based on two parameters nonlinear fitting method. Figure 8 shows the pressure measurement results of EFPI-FBG based sensor at different temperatures without temperature compensation calculation. Figure 9 shows the pressure measurement results at different temperatures with temperature compensation calculation. One can see that the influence of temperature has been greatly reduced.

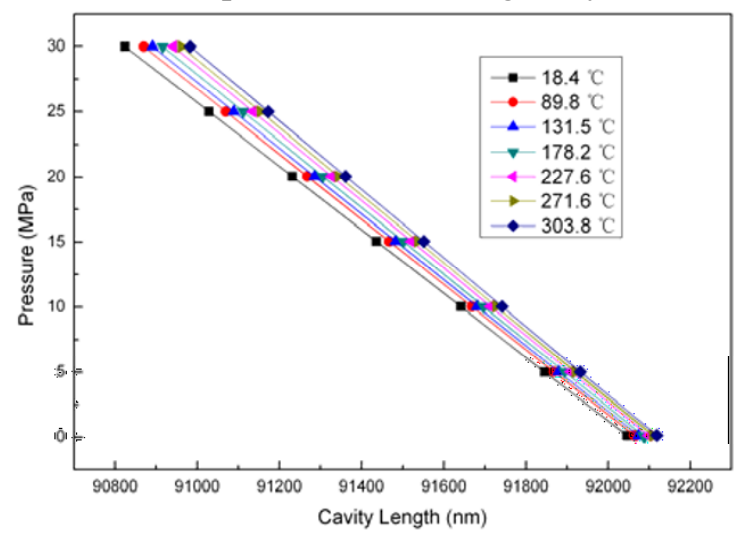

Fig. 8 Pressure measurement results at different temperatures without temperature compensation calculation.

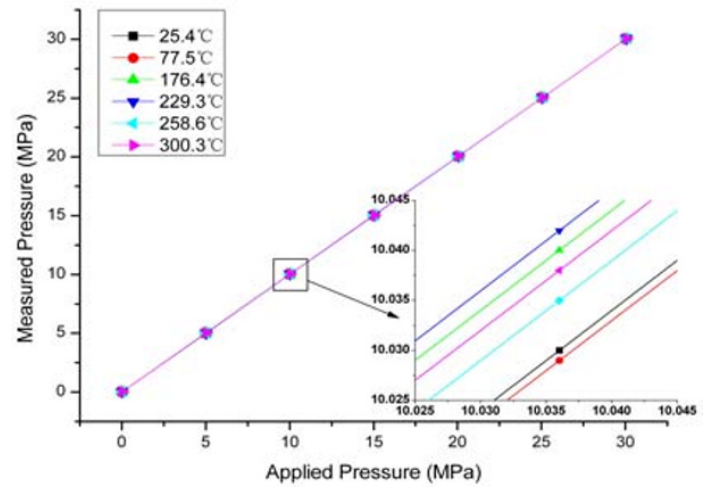

Fig. 9 Pressure measurement results at different temperatures with temperature compensation calculation. 
Long-term stability of EFPI-based sensor operating in high temperature has also been investigated. Experiment research results show that the stability of EFPI sensor is mainly affected by the residual stress induced by $\mathrm{CO}_{2}$ laser thermal bonding process. The release of residual stress leads to the drift of EFPI sensor. To solve this problem, an annealing treatment is done before EFPI-based sensor is applied for pressure measurement. Figure 10 shows the influence of residual stress release on EFPI cavity length at $700{ }^{\circ} \mathrm{C}$. We can see that the residual stress has been released up to $90 \%$ after 15 day's annealing. After annealing treatment, EFPI-based sensor exhibits a good long-term stability. Figure 11 shows the measurement result of the long-term stability of two EFPI-based sensors in $300{ }^{\circ} \mathrm{C}$ within six months. From Fig. 11 one can see that the long term drift is less than $0.1 \%$ in full scale of $35 \mathrm{~Pa}$.

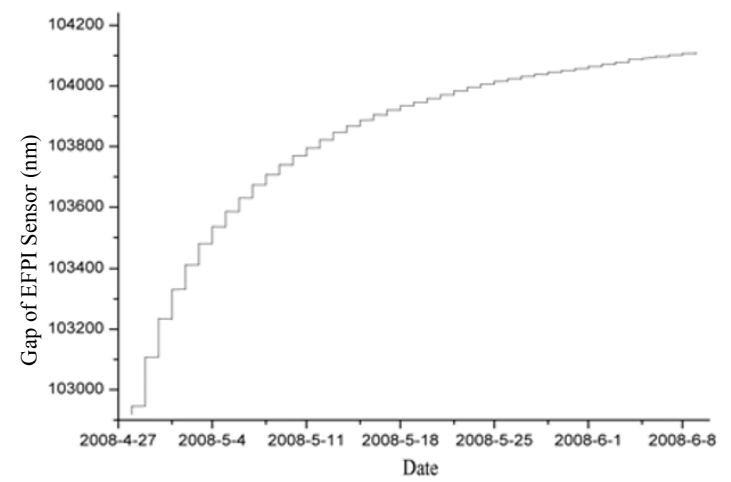

Fig. 10 Influence of residual stress release on EFPI cavity length at $700{ }^{\circ} \mathrm{C}$.

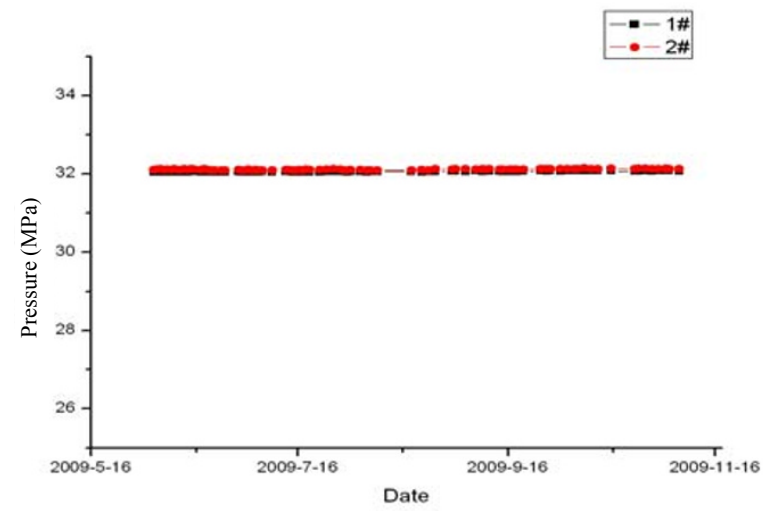

Fig. 11 Measurement result of the long-term stability of two EFPI-based sensors in $300{ }^{\circ} \mathrm{C}$.
In order to improve the measurement resolution of EFPI cavity length and expand the measurement range, Qi Wang et al. [49] developed a broadband optical fiber sensor interrogator system, in which a widely tunable erbium-doped fiber ring laser with a tunning range over $145 \mathrm{~nm}$ was used. Demodulating with this system, EFPI-FBG multiplexed sensor exhibits better performance of resolution measurement and stability. Experimental results show that the limit resolution of EFPI cavity length of $0.08 \mathrm{~nm}$ is reached, corresponding to a pressure resolution of $0.32 \mathrm{kPa}$, as shown in Fig. 12. And the resolution of FBG wavelength is $0.63 \mathrm{pm}$, corresponding to the temperature resolution of $0.065{ }^{\circ} \mathrm{C}$, as shown in Fig. 13.

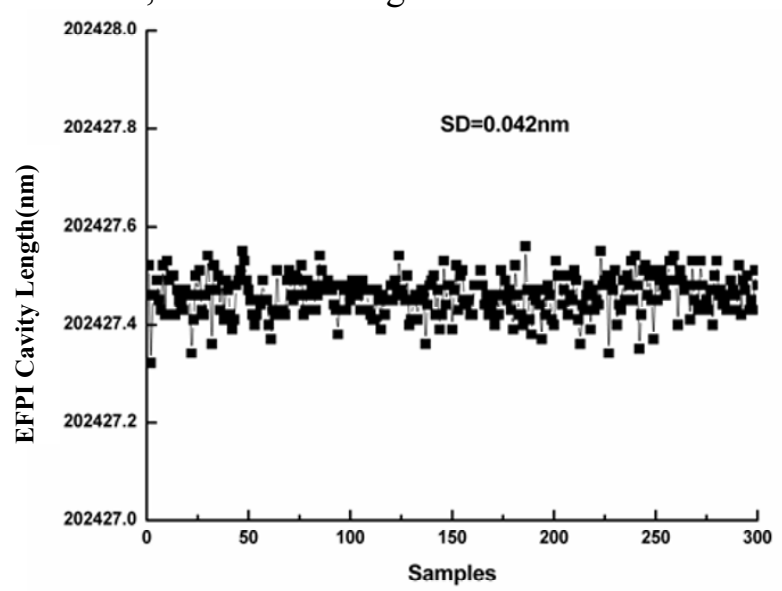

Fig. 12 Resolution measurement of EFPI cavity length.

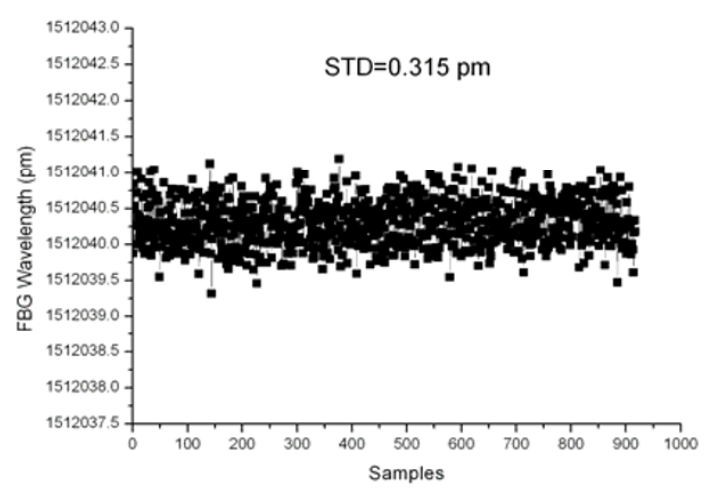

Fig. 13 Resolution measurement of FBG wavelength.

\subsection{Liquid-level monitoring}

Due to the advantage of high sensitivity, DEFPI-based sensors have drawn great interests for 
low pressure measurements, such as the liquid-level monitoring [50, 51]. However, the temperature dependence of DEFPI-based optical fiber sensor induced by trapped air expansion and thermal expansion coefficient mismatch of the material in the sensor construction will cause a larger pressure error.

To solve this problem, Qiaoyun Wang [52] et al. proposed an all silica DEFPI-based optical fiber differential pressure sensor with a pressure balance structure, schematically shown in Fig. 14. A capillary with cone-shaped cup and two holes is employed as the alignment and support component of DEFPI-based sensor. Due to vent structure and low thermal expansion coefficient of silica material, this sensor has low temperature response and high sensitivity. A sensitivity of $25.89 \mathrm{~nm} / \mathrm{kPa}$ in the range of $0-3 \mathrm{kPa}$ with a linear correlation coefficient of 0.99958 has been obtained, as shown in Fig. 15. And a resolution of $0.12 \mathrm{~nm}$, corresponding to a pressure resolution of $4.7 \mathrm{~Pa}$, has been achieved.

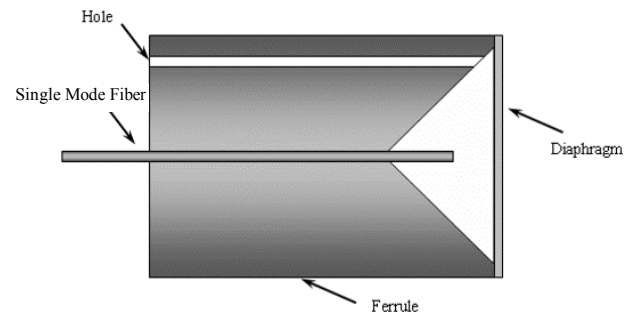

Fig. 14 Structure of diaphragm-based optical fiber pressure sensor.

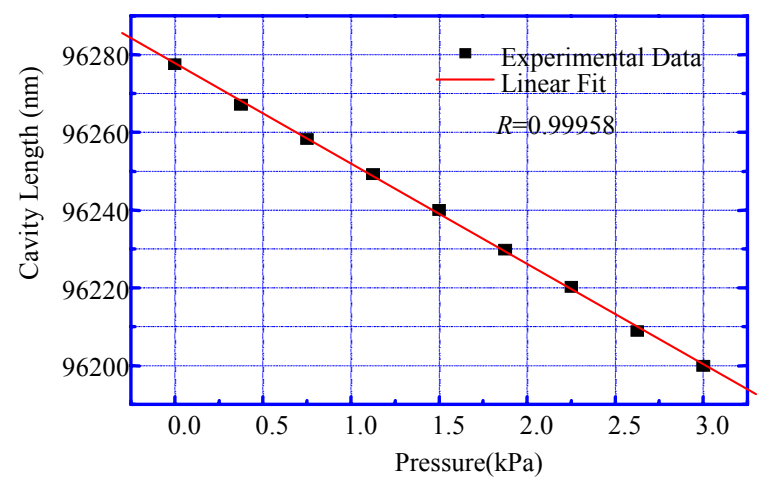

Fig. 15 Pressure response of DEFPI-based sensor at room temperature.

\subsection{Acoustic wave detection}

In recent years, DEFPI-based fiber optic sensors have become a hot topic in the acoustic signal detection due to its high sensitivity, wide frequency response, and immunity to electromagnetic interference. Various kinds of DEFPI-based fiber optic sensors, such as silica diaphragm spliced with silica capillary [53, 54], glass or silicon diaphragm bonded to silicon base [55], and dipped polymer membrane on hollow fiber tip [22] have been developed and applied in the detection of hydroacoustic waves and partial discharge in power transformer [56]. However, most of these sensors can not suit for aeroacoustic detection due to the mismatch of acoustic impedance with the sensor's diaphragm.

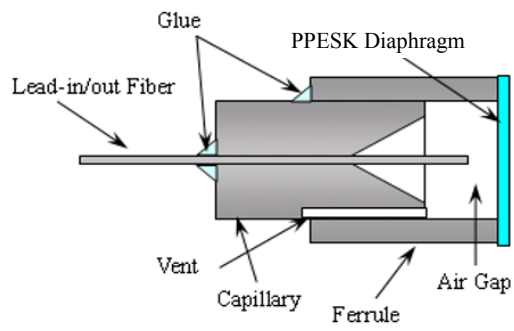

Fig. 16 Configuration of DEFPI-based fiber optic acoustic sensor.

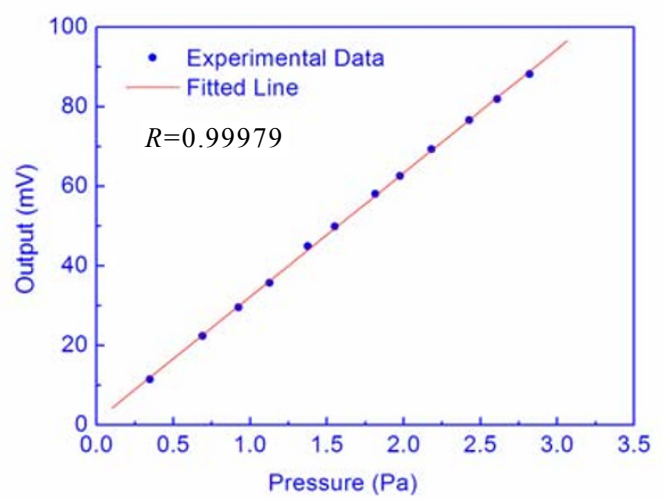

Fig. 17 Linearity between output of DEFPI-based acoustic sensor and sound pressure at $1 \mathrm{kHz}$.

To meet the needs of aeroacoustic detection, a novel polymer poly (phthalazinone ether sulfone ketone, PPESK) diaphragm has been employed as the sensing element of DEFPI-based fiber acoustic 
sensor [57], as shown in Fig. 16. Owing to the good mechanical and optical features of PPESK diaphragm and application of the interferometric/ intensity demodulation mechanism, a system sensitivity of $31 \mathrm{mV} / \mathrm{Pa}$ in the frequency range of $100 \mathrm{~Hz}$ to $12.7 \mathrm{kHz}$ and a signal to noise ratio (SNR) of $29 \mathrm{~dB}$ at $1 \mathrm{kHz}$ have been obtained. The linear response of sensor is from $0.35 \mathrm{~Pa}$ to $2.82 \mathrm{~Pa}$, corresponding to $85 \mathrm{~dB}-103 \mathrm{~dB}$ sound pressure level (SPL) (re.20 $\mu \mathrm{Pa})$, as shown in Fig. 17.

\subsection{Photoacoustic spectrometer for trace gas detection}

Trace gas detection plays an important role in many areas, such as environmental atmospheric monitoring, combustion study, plant and insect respiration study, and medical diagnosis [58-61]. Based on near-infrared photoacoustic spectroscopy (PAS), trace gas analysis is carried out by detecting photoacoustic (PA) signal [62-64]. However, conventional electric acoustic sensors (microphone), which are usually used to detect PA signal, are not competent for operation in some extremely harsh environment. For PA signal detecting in harsh environment, an all-optical PAS system based on DEFPI fiber optic acoustic sensor (as shown in Fig. 16) for trace gas detection at atmospheric pressure and room temperature is proposed, schematically shown in Fig. 18.

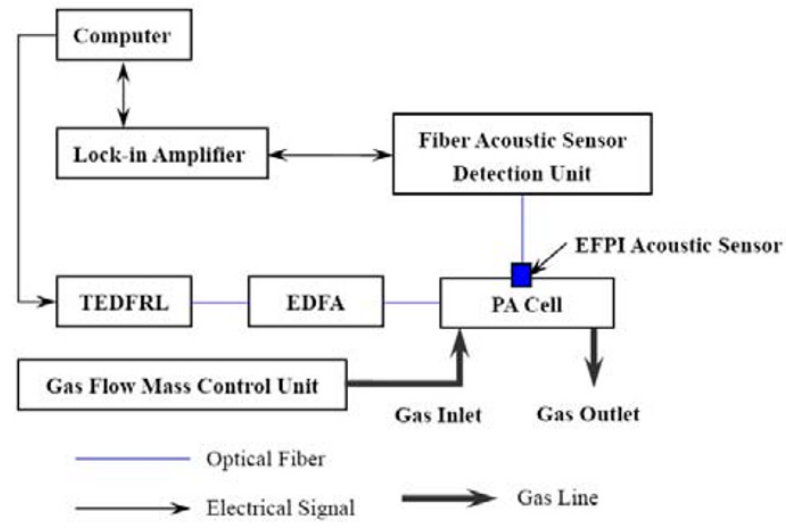

Fig. 18 Configuration of the all-optical PAS system.

Taking the advantages of the wavelength modulation and second harmonic detection method, the all-optical PAS system is demonstrated by detecting the acetylene at room temperature and atmospheric pressure. Experimental results show a good linear relationship between PA signal and the acetylene concentration in the range of $0.05 \mathrm{ppm}$ to $1 \mathrm{ppm}$ with a linear correlation coefficient of 0.99981 , as shown in Fig. 19. And a minimum detectable limit of $1.56 \mathrm{ppb}$ has been achieved.

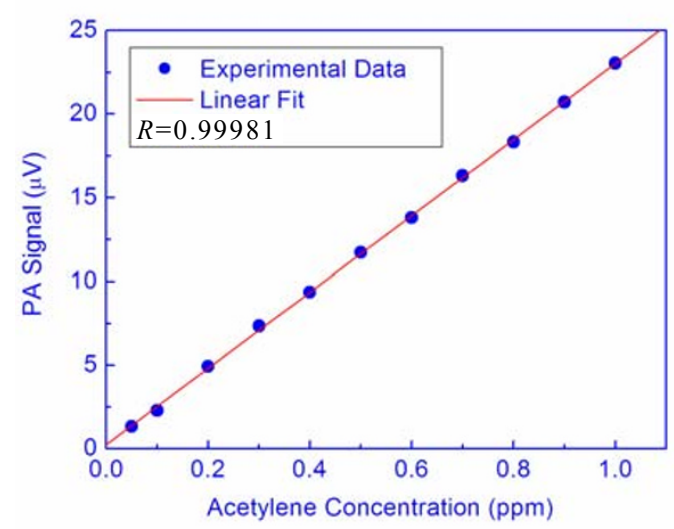

Fig. 19 Linear response of PA signals to acetylene concentration at room temperature and atmosphere pressure.

\section{Conclusions}

The present paper is devoted to a comprehensive introduction to fiber EFPI-based pressure sensors. In the past two decades, EFPI-based pressure sensors have undergone a significant growth and substantial research has been carried out on it. Signal demodulation algorithm based on cross correlation and MSE estimation have been proposed for retrieving the cavity length of EFPI, and a resolution up to $0.08 \mathrm{~nm}$ and absolute measurement over a large dynamic range have been implemented. For downhole monitoring, an EFPI and a FBG cascade multiplexing fiber-optic sensor system has been developed, which can operate in the temperature up to $300{ }^{\circ} \mathrm{C}$ with good long-term stability and extremely low temperature-pressure cross-sensitivity. Diaphragm-based EFPI sensors have been successfully used for low pressure and acoustic wave detection. Experimental results show that a 
sensitivity of $31 \mathrm{mV} / \mathrm{Pa}$ in the frequency range from $100 \mathrm{~Hz}$ to $12.7 \mathrm{kHz}$ for aeroacoustic wave detection has been obtained. Applying fiber optical acoustic sensor to photoacoustic spectrometer, an all-optical PAS system is realized recently.

We wish this paper would provide the researchers some useful information regarding EFPI-based pressure sensors and encourage them to take this area for further research and development.

\section{References}

[1] Robert J. Schroeder, Rogerio T. Ramos, and Tsutomu Yamate, "Fiber optic sensors for oil field services," in Proc. Fiber Optic Sensor Technology and Applications, Boston, pp. 12-22, 1999.

[2] Alan D. Kersey and F. K. Didden, "CiDRA: leveraging mulitchannel telecommunications technology for enhanced downhole monitoring capabilities in the oil and gas industry," in Proc. Fiber Optic Sensor Technology and Applications, Boston, pp. 12-22, 1999.

[3] Brian Culshaw, W. Craig Michie, and Peter T. Gardiner, "Smart structures: the role of fiber optics," in Proc. Interferometry'94: Interferometric Fiber Sensing, Warsaw, pp. 134-151, 1994.

[4]A. Wang, H. Xiao, Russell G. May, J. Wang, W. Zhao, and J. Deng, "Optical fiber sensors for harsh environments," in International Conference on Sensors and Control Techniques, Wuhan, pp. 2-6, 2000.

[5] S. H. Aref, H. Latifi, M. I. Zibaii, et al., "Fiber optic Fabry Perot pressure sensor with low sensitivity to temperature changes for downhole application," Optics Communications, vol. 269, no. 2, pp. 322-330, 2007.

[6] S. H. Aref, M. I. Zibaii, and H. Latifi. "An improved fiber optic pressure and temperature sensor for downhole application," Meas. Sci. Technol., vol. 20, no. 3, pp. 1-6, 2009.

[7] J. W. Berthold, "Historical review of microbend fiber-optic sensors,' J. Lightwave Technol., vol. 13, no. 7, pp. 1193-1199, 1995.

[8] W. B. Spillman, "Multimode fiber-optic pressure sensor based on the photoelastic effect," Opt. Lett., vol. 7, no. 8, pp. 388-390, 1982.

[9] I. P. Giles, S. McNeill, and B. Culshaw, "A stable remote intensity based fiber sensor,' J. Phys., vol. 18, no. 6, pp. 1124-1126, 1985.

[10] A. Wang, S. He, X. Fang, X. Jin, and J. Lin, "Optical fiber pressure sensor based on photoelastic effect and its applications," J. Lightwave Technol., vol. 10, no. 10, pp. 1466-1472, 1992.
[11] D. J. Hill and G. A. Cranch, "Gain in hydrostatic pressure sensitivity of coated fiber Bragg grating," Electron. Lett., vol. 35, no. 15, pp. 1268-1269, 1999.

[12] M. G. Xu, H. Geiger, and J. P. Dakin, “Fiber grating pressure sensor with enhanced sensitivity using a glass-bubble housing,' Electron. Lett., vol. 32, no. 2, pp. 128-129, 1996.

[13] Ph. M. Nellen, P. Mauron, A. Frank, U. Sennhauser, K. Bohnert, P. Pequignot, P. Bodor, and H. Brändle, "Reliability of fiber Bragg grating based sensors for downhole applications," Sens. Actuators A: Phys., vol. 103, no. 13, pp. 364-376, 2003.

[14] K. A. Murphy, Michael F. Gunther, Ashish M. Vengsarkar, and Richard O.Claus, "Quadrature phase-shifted extrinsic Fabry-Perot optical fiber sensors," Opt. Lett., vol. 24, no. 6, pp. 273-275, 1991.

[15] K. A. Murphy, M. F. Gunther, R. G. May, R. O. Claus, T. A. Tran, J. A. Greene, and P. G. Duncan, "EFPI sensor manufacturing and applications," in Proc. Smart Structures and Materials 1996: Industrial and Commercial Applications, San Diego, pp. 476-482, 2005.

[16] A. Wang, "Optical fiber sensors for energy-production and energy-intensive industries," in Proc. the International Society for Optical Engineering, Shanghai, pp. 377-381, 2002.

[17] J. Deng, H. Xiao, W. Huo, et al., "Optical fiber sensor-based detection of Partial discharges in power transformers," Optics \& Laser Technology, vol. 33, no. 5, pp. 305-311, 2001.

[18] J. Xu, G. Piekrell, and B. Yu, "Epoxy-free high temperature fiber optic pressure sensors for gas turbine engine applications," in Proc. Sensors for Harsh Environments, Philadelphia, pp. 1-10, 2004.

[19] G. C. Hill, R. Melamud, F. E. Declercq, et al., "SU-8MEMS Fabry-Perot pressure sensor," Sens. Actuators A: Phys., vol. 138, no. 1, pp. 52-62, 2007.

[20] J. Zhou, S. Dasgupta, H. Kobayashi, J. M. Wolff, H. E. Jackson, and J. T. Boyd, "Optically interrogated MEMS pressure sensors for propulsion applications," Opt. Eng., vol. 40, no. 4, pp. 598-604, 2001.

[21] C. Yang, C. Zhao, Lester Wold, and Kenton R. Kaufman, "Biocompatibility of a physiological pressure sensor," Biosensors and Bioelectronics, vol. 19, no. 1, pp. 51-58, 2003.

[22] Edvard Cibula and Denis Donlagic, "Miniature fiber-optic pressure sensor with a polymer diaphragm," Appl. Opt., vol. 44, no. 14, pp. 2736-2744, 2005.

[23] Zhu Yizheng, G.Pickrell, Wang Xinwei, et al., "Miniature fiber optic pressure sensor for turbine engines," in Proc. Sensors for harsh Environments, Bellingham, pp. 11-18, 2004. 
[24] Kentaro Totsu, Yoichi Haga, and Masayoshi Esashi, "Ultra-miniature fiber-optic pressure sensor using white light interferometry," J. Micromech. Microeng., vol. 15, no. 1, pp. 71-75, 2005.

[25] GE Yi-xian, WANG Ming, CHEN Xu-xing, and LI Ming, "A Novel Fabry-Perot MEMS Fiber Pressure Sensor Based on Intensity Demodulation Method Interferometry," Chinese Journal of Sensors and Actuators, vol. 19, no. 3, pp. 1832-1839, 2006(in Chinese).

[26] V. Arya, M. D. Vries, K. A. Murphy, A. Wang, and R. O. Claus, "Exact analysis of the extrinsic Fabry-Pérot interferometric optical fiber sensor using Kirchhoff's diffraction formalism," Opt. Fiber Technol., vol. 1, no. 4, pp. 380-384, 1995.

[27] C. E. Lee and H. F. Taylor, "Fiber-optic Fabry-Pérot temperature sensor using a low-coherence source," J. Lightw. Technol., vol. 9, no. 1, pp. 129-134, 1991.

[28] Y. N. Ning, K. T. V. Grattan, and A. W. Palmer, "Fibre-optic interferometric systems using low-coherent light sources," Sens. Actuators A: Phys., vol. 30, no. 3, pp. 181-192, 1992.

[29] Y. J. Rao and D. A. Jackson, "Recent progress in fibre optic low-coherence interferometry," Meas. Sci. Technol., vol. 7, no. 7, pp. 981-999, 1996.

[30] F. Shen and A. Wang, "Frequency-estimation-based signal-processing algorithm for white-light optical fiber Fabry-Perot interferometers," Appl. Opt., vol. 44, no. 25, pp. 5206-5214, 2005.

[31] Y. J. Rao, "Demodulation algorithm for spatial-frequency-division-multiplexed fiber-optic Fizeau strain sensor networks," Opt. Lett., vol. 31, no. 6, pp. 700-702, 2006.

[32] Jiang Y., "Fourier transform white-light interferometry for the measurement of fiber-optic Fabry-Perot interferometric sensors," IEEE Photonics Technol. Lett., vol. 20, no. 2, pp. 75-77, 2008.

[33] M. Han, "Theoretical and Experimental Study of Low-Finesse Extrinsic Fabry-Perot Interferometric Fiber Optic Sensors," Ph. D. dissertation, Electrical and Computer Engineering, Virginia Tech., USA, 2006.

[34] A. Wang, H. Xiao, J. Wang, Z. Wang, W. Zhao, and R. G. May, "Self-calibrated interferometric-intensity based optical fiber sensors," J. Lightw. Technol., vol. 19, no. 10, pp. 1495-1501, 2001.

[35] Bing Yu, Anbo Wang, and Gary R. Pickrell, "Analysis of Fiber Fabry-Pérot Interferometric Sensors Using Low-Coherence Light Sources," J. Lightw. Technol., vol. 24, no 4, pp. 1758-1767, 2006.

[36] R. G. May, A. Wang, H. Xiao, et al., "SCIIB pressure sensors for oil extraction applications," in Proc. Harsh Environment Sensors II, Boston, MA, pp. 29-35, 1999.

[37] Guiju Zhang, Qingxu Yu, and Shide Song, "An investigation of interference/intensity demodulated fiber optic Fabry-Perot cavity sensor," Sens. Actuators A: Phys., vol. 116, no. 1, pp. 33-38, 2005.

[38] P. C. Beard and T. N. Mills, "Extrinsic optical-fiber ultrasound sensor using a thin polymer film as a low-finesse Fabry-Pérot interferometer," Appl. Opt., vol. 35 , no. 4 , pp. $663-675,1996$.

[39] J. F. Dorighi, S. Krishnaswamy, and J. Achenbach, "Stabilization of an embedded fiber optic Fabry-Pérot sensor for ultra-sound detection," IEEE Trans. Ultrason. Ferroelectr. and Freq. Control, vol. 42, no. 5, pp. 820-824, 1995.

[40] J. Xu, X. Wang, K. L. Cooper, et al., "Miniature all-silica fiber optic pressure and acoustic sensors," Opt. Lett., vol. 30, no. 24, pp. 3269-3271, 2005.

[41] N. Fürstenau, M. Schmidt, H. Horack, W. Goetze, and W. Schmidt, "Extrinsic Fabry-Pérot interferometer vibration and acoustic systems for airport ground traffic monitoring," in Proc. Inst. Elect. Eng. -Optoelectron., vol. 144, no. 3, pp. 134-144, 1997.

[42] B. Yu, D. W. Kim, J. Deng, H. Xiao, and A. Wang, "Fiber Fabry-Pérot sensors for partial discharge detection in power transformers," Appl. Opt., vol. 42, no. 16, pp. 3241-3250, 2003.

[43] B. Yu and A. Wang, "Grating-assisted demodulation of interferometric optical sensors," Appl. Opt., vol. 42, no. 34, pp. 6824-6829, 2003.

[44] H. Xiao, J. D. Deng, G. Pickrell, R. G.May, and A.Wang, "Single-crystal sapphire fiber-based strain sensor for high temperature applications," J. Lightw. Technol., vol. 21, no. 10, pp. 2276-2283, 2003.

[45]S. A. Egorov, A. N. Mamaev, I. G. Likhachiev, Y. A. Ershov, A. S. Voloshin, and E. Nir, "Advanced signal processing method for interferometric fiber-optic sensors with straightforward spectral detection," in Proc. Sensors and controls for advanced manufacturing, Pittsburgh PA, pp. 44-48, 1997.

[46] Jing Zhenguo and Yu Qingxu, "White light optical fiber EFPI sensor based on cross-correlation signal processing method," in Proc. Test and Measurement, pp. 3509-3511, 2005.

[47] Shide Song, "Study on the Characteristics and Sensing Applications of Long Period Fiber Gratings," Ph. D. dissertation, Dalian University of Technology, China, 2006.

[48]Qi Wang, Lei Zhang, Changsen Sun, and Qingxu Yu, "Multiplexed Fiber-Optic Pressure and Temperature Sensor System for Down-Hole Measurement," IEEE Sensors Journal, vol. 8, no. 11, pp. 1879-1883, 2008.

[49] Qi Wang, "Study on Key Technologies of Fiber 
EFPI/FBG Sensing System for Oil Well Logging," $\mathrm{Ph}$. D. dissertation, Dalian University of Technology, China, 2009.

[50] Tao Lü and Suping Yang, "Extrinsic Fabry-Perot cavity optical fiber liquid-level sensor," Appl. Opt., vol. 46, no. 18, pp. 3862-3867, 2007.

[51] Tao Lü, Zhengjia Li, Danqing Xia, Kaihua He, and Guangyong Zhang, "Asymmetric Fabry-Perot fiber-optic pressure sensor for liquid-level measurement," Review of Scientific Instruments, vol. 80, no. 3, pp. 033104, 2009.

[52] Qiaoyun Wang, Wenhua Wang, Xinsheng Jiang, and Qingxu Yu, "Diaphragm-based Extrinsic Fabry-Perot Interferometric optical fiber pressure sensor," presented at Proc. Advanced Optical Manufacturing and Testing Technologies, Dalian, China, 2010.

[53] D. Donlagic and E. Cibula, "All-fiber high-sensitivity pressure sensor with $\mathrm{SiO}_{2}$ diaphragm," Opt. Lett., vol. 30, no. 16, pp. 2071-2073, 2005.

[54] Y. Zhu and A. Wang, "Miniature fiber-optic pressure sensor", IEEE Photo. Technol. Lett., vol. 17, no. 2, pp. 447-449, 2005.

[55]D. C. Abeysinghe, S. Dasgupta, J. T. Boyd, and H. E. Jackson, "A novel MEMS pressure sensor fabricated on an optical fiber," IEEE Photon. Technol. Lett., vol. 13, no. 9, pp. 993-995, 2001.

[56] X. Wang, B. Li, Z. Xiao, et al., "An ultra-sensitive optical MEMS sensor for partial discharge detection," J. Micromech. Microeng., vol. 15, no. 3, pp. 521-527, 2005.

[57] Qiaoyun Wang and Qingxu Yu, "Polymer diaphragm based sensitive fiber optic Fabry-Perot acoustic sensor," Chinese Optics Letters, vol. 8, no.
3, pp. 266-269, 2010(in Chinese).

[58] P. Martin, "Near-infrared diode laser spectroscopy in chemical process and environmental air monitoring," Chemical Society Reviews, vol. 31, no. 4, pp. 201-210, 2002.

[59] M. Sigrist, R. Bartlome, D. Marinov, J. Rey, D. Vogler, and H. W chter, "Trace gas monitoring with infrared laser-based detection schemes," Applied Physics B: Lasers and Optics, vol. 90, no, 2, pp.289-300, 2008.

[60] M. van Herpen, A. Ngai, S. Bisson, J. Hackstein, E. Woltering, and F. Harren, "Optical parametric oscillator-based photoacoustic detection of $\mathrm{CO}_{2}$ at $4.23 \mu \mathrm{m}$ allows real-time monitoring of the respiration of small insects," Applied Physics B: Lasers and Optics, vol. 82, no. 4, pp. 665-669, 2006.

[61] M. Pushkarsky, I. Dunayevskiy, M. Prasanna, A. Tsekoun, R. Go, and C. Patel, "High-sensitivity detection of TNT," in Proceedings of the National Academy of Sciences, vol. 103, no. 52, pp. 19630-19634, 2006.

[62] J. Li, X. Gao, L. Fang, W. Zhang, and H. Cha, "Resonant photoacoustic detection of trace gas with DFB diode laser," Optics \& Laser Technology, vol. 39, no. 6, pp. 1144-1149, 2007.

[63] A. Thony and M. Sigrist, "New developments in CO2-laser photoacoustic monitoring of trace gases," Infrared Physics \& Technology, vol. 36, no. 2, pp. 585-615, 1995.

[64] Y. Peng, W. Zhang, L. Li, and Q. Yu, "Tunable fiber laser and fiber amplifier based photoacoustic spectrometer for trace gas detection," Spectrochimica Acta Part A: Molecular and Biomolecular Spectroscopy, vol. 74, no. 4, pp. 924-927, 2009. 